\title{
Investigating novel biomarkers of immune activation and modulation in the context of sedentary behaviour: a multicentre prospective ischemic stroke cohort study
}

Katinka Nordheim Alme ${ }^{1,2^{*}}$, Torunn Askim³ ${ }^{3}$ Jörg Assmus ${ }^{4}$, Tom Eirik Mollnes ${ }^{5,6,7}$, Mala Naik ${ }^{2,8}$, Halvor Næss ${ }^{1,9,10}$, Ingvild Saltvedt ${ }^{3,11}$, Per-Magne Ueland ${ }^{12}$, Arve Ulvik ${ }^{12}$ and Anne-Brita Knapskog ${ }^{13}$

\begin{abstract}
Background: Sedentary behaviour is associated with disease, but the molecular mechanisms are not understood. Valid biomarkers with predictive and explanatory properties are required. Therefore, we have investigated traditional and novel biomarkers of inflammation and immune modulation and their association to objectively measured sedentary behaviour in an ischemic stroke population.

Methods: Patients admitted to hospital with acute ischemic stroke were included in the multicentre Norwegian Cognitive Impairment After Stroke (Nor-COAST) study $(n=815)$. For this sub-study $(n=257)$, sedentary behaviour was registered 3 months after stroke using position transition data from the body-worn sensor, ActivPal ${ }^{\oplus}$. Blood samples were analysed for high sensitive C-reactive protein (hsCRP), the cytokines interleukin-6 (IL-6) and 10 (IL-10), neopterin, tryptophan (Trp), kynurenine (kyn), kynurenic acid (KA), and three B6 vitamers, pyridoxal 5'-phosphate $(P L P)$, pyridoxal $(P L)$, and pyridoxic acid (PA). The kynurenine/tryptophan ratio (KTR) and the pyridoxic acid ratio index (PAr $=P A: P L+P L P)$ were calculated.

Results: Of the 815 patients included in the main study, 700 attended the three-month follow-up, and 257 fulfilled the inclusion criteria for this study. Sedentary time was significantly associated with levels of hsCRP, IL-6, neopterin, PAr-index, and KA adjusted for age, sex, waist circumference, and creatinine. In a fully adjusted model including all the significant biomarkers except hsCRP (because of missing values), sedentary time was independently positively associated with the PAr-index and negatively with KA. We did not find an association between sedentary behaviour, IL-10, and KTR.

Conclusions: The PAr-index is known to capture several modes of inflammation and has previously shown predictive abilities for future stroke. This novel result indicates that the PAr-index could be a useful biomarker in future studies on sedentary behaviour and disease progression. KA is an important modulator of inflammation, and this finding opens new and exciting pathways to understand the hazards of sedentary behaviour.
\end{abstract}

\footnotetext{
* Correspondence: katinka.alme@gmail.com

'Institute of Clinical Medicine (K1), University of Bergen, Bergen, Norway

${ }^{2}$ Department of Internal Medicine, Haraldsplass Deaconess Hospital, Bergen, Norway

Full list of author information is available at the end of the article
} 
Trial registration: The study was registered at Clinicaltrials.gov (NCT02650531). First posted 08/01/2016.

Keywords: Sedentary behavior, Inflammation, Immune modulation, Vascular disease, Kynurenine pathway, Stroke

\section{Background}

Sedentary behaviour is associated with an increased risk of vascular disease, amongst others, through inflammatory pathways $[1-5]$. The impact depends on the properties of the sedentary behaviour and the corresponding physical activity, which can vary in bout length, intensity, and habit [1, 6-9]. To increase the understanding of the link between sedentary behaviour and vascular disease, valid biomarkers with predictive and explanatory properties are required.

Cytokines can be predominantly pro- or antiinflammatory, but their downstream effect also depends on other contextual factors. Also, cytokines vary in their biological and analytical properties, and some of them, such as interleukin $1 \beta$ (IL-1 $\beta$ ) and interferon $\gamma$ (IFN- $\gamma$ ), are therefore often measured indirectly by other more stable downstream molecules $[5,10,11]$. The inflammatory biomarker C-reactive protein (CRP) is a downstream marker of interleukin 6 (IL-6) and IL-1 $\beta$ [10]. The inflammation associated with this pathway has been shown to increase with age and to be associated with sedentary behaviour and disease development $[1,2,10$, 12-14]. Interleukin-10 (IL-10) is a predominantly antiinflammatory cytokine found to be induced by acute bouts of moderate to vigorous physical activity (MVPA) and in response to chronic exercise [4, 15-17]. The association to sedentary behaviour is not clear.

The inflammatory pathway associated with IFN-y can be measured by the ratio between kynurenine and tryptophan-the kynurenine tryptophan ratio (KTR) - and neopterin secreted by activated macrophages. Kynurenine is a metabolite of the amino acid tryptophan and is the first metabolic step of the kynurenine pathway (KP) $[18,19]$. KTR and neopterin have been shown to predict future coronary events $[11,20]$, but the association to sedentary behaviour is unclear $[21,22]$.

A novel and sensitive biomarker of inflammation, the pyridoxal acid ratio index (PAr-index $=($ pyridoxic acid (PA): (pyridoxal (PL) + pyridoxal 5-phosphate (PLP)), represents several modes of inflammation and captures the effect of the inflammatory pathways associated with CRP, white blood cell count, neopterin, and KTR [23, 24]. The PAr-index has been shown to predict future stroke with better precision than high sensitive Creactive protein (hsCRP) [25] but has never been studied in the context of sedentary behaviour.

Kynurenic acid (KA), a side-product in the KP, is not an inflammatory biomarker but part of a negative feedback mechanism inducing immune tolerance $[26,27]$.
The role of KA in health and disease is not fully understood [26] and KA has never been investigated in relation to sedentary behaviour. KA has been found to increase following physical exercise and KA can potentially be one of the molecular links between sedentary behaviour and inflammation [22, 28-32].

The primary objective of the present study was to investigate the association between novel biomarkers of inflammation and immune modulation and objectively measured habitual sedentary behaviour in a stroke population. The secondary objective was to investigate the impact of bout duration on these associations. Our hypothesis was that sedentary behaviour would be positively associated with a pro-inflammatory profile.

\section{Material and methods}

\section{Subjects}

The prospective cohort study, the Norwegian Cognitive Impairment After Stroke Study (Nor-COAST), included 815 adults admitted for acute stroke at one of five contributing hospitals from May 2015 through March 2017. Details of the Nor-COAST study, including inclusion and exclusion criteria and patient selection, have been published elsewhere [33, 34]. In the current study, only patients with ischemic stroke who attended the threemonth follow-up, had blood samples drawn for direct analyses at the local laboratory and for storage in the bio-bank, were able to walk $50 \mathrm{~m}$ independently or with support from a person/walker (Barthel index item 9, $\geq 10$ points), and had valid activity data were included.

\section{Clinical data and laboratory analyses}

Demographic information, as well as information about risk factors of vascular disease, stroke severity, and functional outcomes were collected during the index stay and at the three-month follow-up. Stroke severity was measured by the National Institute of Health Stroke Scale (NIHSS) at the time of hospital arrival and at three-month follow-up [35]. Functional state was measured by the Barthel index and the modified Rankin scale at discharge or day seven. The Barthel index is a 10-item assessment for basal activities of daily living, with a maximum score of 100 points indicating no functional deficits [36]. The modified Rankin scale is a fivepoint scale measuring global disability, where $0-2$ is defined as 'good outcomes', 3-5 indicates increasingly disability, and 6 is death [37]. 


\section{Laboratory markers}

Non-fasting blood samples were collected at the threemonth follow-up at the outpatient clinic.

Routine clinical-chemical analyses The blood samples were analysed for high sensitive C-reactive protein (hsCRP, $\mathrm{mg} / \mathrm{L}$ ) and creatinine $(\mu \mathrm{mol} / \mathrm{L})$ at the local laboratory directly. The estimated glomerular filtration rate (eGFR) was calculated with the Chronic Kidney Disease Epidemiology Collaboration formula [38] using a dedicated STATA software package.

Sample collection of research analyses Aliquots of serum and plasma were immediately frozen at $-80^{\circ} \mathrm{C}$ at the inclusion hospital. The samples were transported on dry ice and stored at BioBank1, Central Norway Health Authority. In 2019, two aliquots of plasma were transported on dry ice to the Research Laboratory Nordland Hospital (Bodø, Norway) and Bevital A/S (Bergen, Norway), respectively, and were thawed only once.

Cytokines Interleukin (IL)-6 and IL-10 were measured as the classical cytokines previously shown to be of interest in these patients (see Introduction). They were analysed at the Research Laboratory Nordland Hospital using the Bio-Plex technology kits obtained from BioRad Laboratories Inc., Hercules, CA. The assay was performed according to the manufacturer's procedure. Values below the lower detecting limit ( $n=36$ for IL-10, none for IL-6) were replaced by random values below the lower detecting limit when statistics were performed.

Other biomarkers The three vitamin B6 forms pyridoxal 5'-phosphate (PLP), pyridoxal (PL), and 4pyridoxic acid (PA) were measured along with tryptophan, the two tryptophan metabolites kynurenine and kynurenic acid (KA), and neopterin as novel biomarkers of interest (see Introduction) as part of analytic platform $\mathrm{D}$ at Bevital A/S (Bergen, Norway) by liquid chromatography/tandem mass spectrometry using EDTA-plasma. The PAr-index was calculated as PA:(PL + PLP) [23].

\section{Sedentary behaviour}

Sedentary behaviour was defined as sitting or lying. Time in sedentary behaviour was given as total time per day and as time accumulated through pre-defined boutlength categories (<30 min, 30-59 min, 60-89 min, and $\geq 90 \mathrm{~min}$ ). Sedentary behaviour was measured by registering position transition with a single thigh-worn sensor (ActivPal3 ${ }^{\circ}$, Model 20.2, PAL Technologies Ltd., Glasgow, United Kingdom); in patients with hemiparesis, the sensor was attached to the unaffected side. The monitor was attached when the patient visited the outpatient clinic and returned by mail.The threshold for noise was $1.5 \mathrm{~s}$, and sedentary events were merged if they were interrupted by events lasting $\leq 3 \mathrm{~s}$. Measurements were performed for seven consecutive days/nights and analysed using a custom-made MATLAB $^{\circ}$ script (version R2016b, MathWorks, Natick, MA, USA) to extract periods of sedentary behaviour from the recordings. The recordings were analysed for daytime sedentary behaviour, defined as occurring from 08:00 am through 10: $00 \mathrm{pm}$, and a registered period of sedentary behavioura sedentary bout-was divided into two periods if it crossed these time boundaries. Only patients with recordings from at least four full 24-h periods starting from 08:00 am were included. As a final control, manual inspection of the output to identify non-wear time was performed.

\section{Statistics}

Laboratory results and characteristics at baseline and three months were given in means and standard deviations, numbers with percentages, and medians with interquartile range. Differences between groups were tested with the chi-squared test, t-test, and WilcoxonMann-Whitney test.

Sedentary behaviour was given as a continuous variable of mean hours per day, both as total time of sedentary behaviour and as time accumulated through different bout-length categories $(<30 \mathrm{~min}, 30-59 \mathrm{~min}$, 60-89 $\mathrm{min}$, and $\geq 90 \mathrm{~min}$ ).

Associations between variables were assessed using multiple linear and Tobit regressions using the biomarker as the dependent variable and sedentary behaviour as the independent variable adjusted for age, sex, waist circumference, and kidney function [27, 39-41]. The results were given as standardised beta coefficients with confidence intervals and $p$-values. One final regression was made to investigate the independent association between the biomarkers significant in the initial analyses and sedentary behaviour. Here sedentary behaviour was used as the dependent variable, while age, sex, waist circumference, and kidney function were independent variables, in addition to the biomarkers with significant results in the primary analyses, with the exception of hsCRP because of missing values (see below). A sensitivity analysis including only those patients with values for hsCRP was added. The residuals of the regressions did not follow a normal distribution, and the value of the biomarkers were log-transformed. For the cytokines, the significance test was performed using a Tobit regression with left censoring [41], applying the highest value amongst the lower detection limits as cut-off (see laboratory analyses for details). The analyses were performed in STATA/SE 16.1.

In the final population, 88 cases had missing values for hsCRP because this analysis was not available at two of 
the inclusion centres; 12 had missing values for waist circumference and 4 for eGFR.

\section{Results}

\section{Patient characteristics and laboratory values}

Out of 700 patients attending three-month follow-up, 257 fulfilled the inclusion criteria (Fig. 1). Of the included patients, 149 (58\%) were men; mean age was 73 $( \pm 11)$ years; they had a mean body mass index (BMI) of $27( \pm 4.3)$ and a waist circumference of $95( \pm 13)$. Compared to the rest of the Nor-COAST population, our subgroup had a lower NIHSS on admission (3.7 (4.6) vs. 5.0 (6.5), $p=0.004)$ and at 3 months (0.7 (1.3) vs. 1.0 (2.0), $p=0.022)$, had more favourable functional outcomes (Barthel index 98 (6.1) vs. 90 (21), $p<0.001$ ) and scores for global disability (modified Rankin scale (1.5 (0.9) vs. $2.0(1.4), \mathrm{p}<0.001)$ at 3 months. There were no differences in the laboratory results between the patient groups, except for the PAr-index, which was higher in included patients $(0.64(0.44,0.91)$ vs. $0.52(0.39,0.73)$, $p=0.005$ ) (Table 1).

\section{Sedentary behaviour}

The patients in our study had a mean total sedentary time of 9.7 (1.9) hours per day. Of the total time spent sedentary, 4.0 (1.1) hours were accumulated through bouts of less than $30 \mathrm{~min}, 2.5$ (0.9) hours through bouts of 30-59 min, 1.4 (0.9) hours through bouts of 60-89 min, and 1.3 (1.5) hours through bouts $\geq 90 \mathrm{~min}$ (Fig. 2).

\section{Regression analyses}

In the adjusted analyses we found hsCRP $(0.25, p=$ 0.001), IL-6 (0.17, $p=0.009)$, neopterin $(0.12, p=0.034)$, and the PAr-index $(0.21, p<0.001)$ to be positively associated with total sedentary time (Table 2). The KA ($0.10, p=0.045)$ showed an inverse association with sedentary time. No significant associations were found for IL-10 and KTR.

In the final model, where all biomarkers significantly associated with sedentary time were added, only the PAr-index $(0.25, p=0.001)$ and KA $(-0.19, p=0.021)$ remained independently associated with total sedentary time (Table 3). In a sensitivity analyses (Table 4) restricted to those with data on hsCRP, only the PArindex $(0.31, \mathrm{p}=0.001)$ were significantly associated. Adding hsCRP to this model did not change the results (numbers not shown).

There was a tendency towards an increased association with longer sedentary bout lengths for hsCRP, IL-6, neopterin, and PAr-index (Supplementary Table 1).

\section{Discussion}

In this study, we have explored novel biomarkers associated with inflammation and immune modulation and their association to objectively measured sedentary time

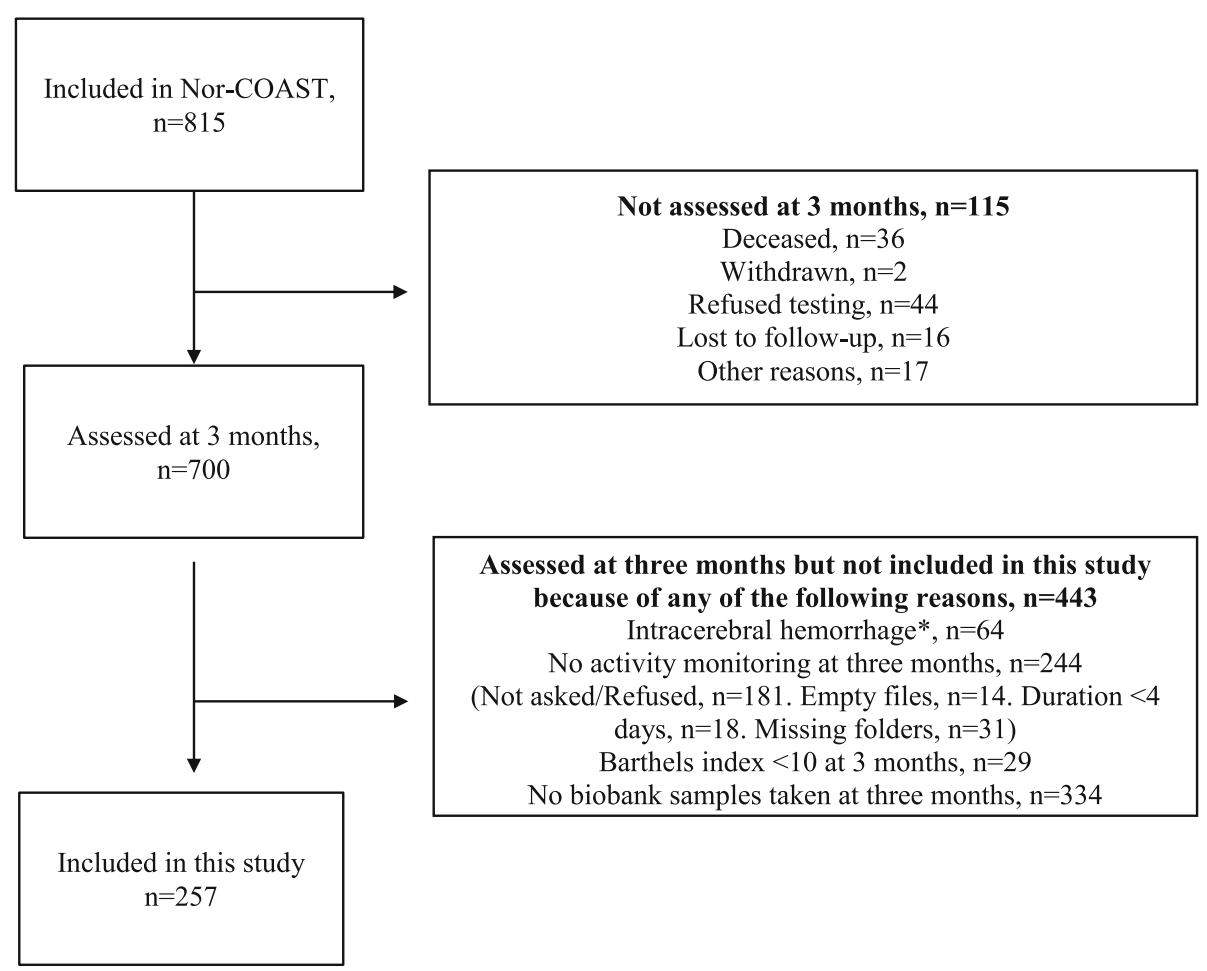

Fig. 1 Patient selection 
Table 1 Baseline and three-month characteristics of the study population compared to the remaining Nor-COAST population

\begin{tabular}{|c|c|c|c|}
\hline & \multicolumn{3}{|l|}{ Total $n=700$} \\
\hline & Not included $(n=443)$ & Included $(n=257)$ & $\mathrm{p}$ \\
\hline Sex, male, n (\%) & $251(57)$ & $149(58)$ & 0.734 \\
\hline Age at three months, mean y (SD) & $74(12)$ & $73(11)$ & 0.162 \\
\hline $\mathrm{BMl}$ at three months, mean $\mathrm{kg} / \mathrm{m}^{2}$ (SD) & $27(4.2)$ & $27(4.3)$ & 0.797 \\
\hline Waist at three months, mean cm (SD) & $97(13)$ & $95(13)$ & 0.283 \\
\hline NIHSS on admission, mean (SD) & $5.0(6.5)$ & $3.7(4.6)$ & 0.004 \\
\hline At three months & $1.0(2.0)$ & $0.7(1.3)$ & 0.022 \\
\hline Modified Rankin scale, mean (SD) & $2.5(1.5)$ & $2.0(1.1)$ & $<0.001$ \\
\hline At three months & $2.0(1.4)$ & $1.5(0.9)$ & $<0.001$ \\
\hline Modified Rankin scale $\leq 2, \mathrm{n}(\%)$ & $269(49)$ & $178(70)$ & $<0.001$ \\
\hline At three months & $304(68)$ & $224(88)$ & $<0.001$ \\
\hline Barthel index, mean (SD) & $79(28)$ & $92(14)$ & $<0.001$ \\
\hline At three months & $90(21)$ & $98(6.1)$ & $<0.001$ \\
\hline \multicolumn{4}{|c|}{ Laboratory values at three months*, median (IQR) } \\
\hline eGFR $(N=272 / 253)$ & $77(60,89)$ & $76(63,88)$ & 0.696 \\
\hline $\operatorname{hsCRP}(N=98 / 169)$ & $1.9(0.7,3.6)$ & $1.6(0.8,3.7)$ & 0.513 \\
\hline IL6 $(N=111 / 257)$ & $4.6(2.5,6.7)$ & $4.6(2.9,7.4)$ & 0.438 \\
\hline $\operatorname{IL} 10(N=111 / 257)$ & $18(8.6,32)$ & $20(8.6,36)$ & 0.304 \\
\hline Neopterin $(N=109 / 257)$ & $16(12,22)$ & $16(12,22)$ & 0.998 \\
\hline PAr-index $(N=109 / 257)$ & $0.52(0.39,0.73)$ & $0.64(0.44,0.91)$ & 0.005 \\
\hline $\operatorname{KTR}(N=109 / 257)$ & $32(28,40)$ & $34(29,42)$ & 0.062 \\
\hline KA $(N=109 / 257)$ & $55(44,75)$ & $59(46,76)$ & 0.276 \\
\hline
\end{tabular}

"Number of patients attending baseline and three months: A total of 558 patients had blood samples taken at three months. Of these, 368 had biobank samples taken. The $\mathrm{N}$ for each individual biomarker is shown for each group (not included/included)

in an ischemic stroke population 3 months after the acute stroke. We have also replicated known associations between traditional biomarkers of inflammation and sedentary behaviour. We found hsCRP, IL-6, neopterin, PAr-index and KA (invers) to be significantly associated with sedentary time. The PAr-index and KA also showed an independent association in a model including IL-6 and neopterin.

The PAr-index has never been studied in the context of sedentary behaviour. The PAr-index reflects several modes of inflammation and has been shown to be associated with CRP, white blood cell count, and markers of cellular immune activation such as neopterin and KTR [23]. In accordance to this, the independent association in the fully adjusted model illustrates that the PAr-index captures the effect of the other pathways by attenuating the association to IL-6 and neopterin when included in the same model. As the PAr-index has recently found to be associated with the risk of future stroke, it might be a valid and clinically relevant biomarker for future intervention studies on sedentary behaviour [25].

The association between sedentary time, hsCRP and IL-6 confirms prior findings, both from observational and intervention studies $[1,2,8,40,42]$. The results from intervention studies imply a causal relationship between sedentary behaviour and inflammation, and Noz et al. found a phenotypic shift in the innate immune system towards a less inflammatory phenotype of circulating monocytes in vitro when sedentary time was replaced with physical activity [1]. Other studies have found a change in the inflammatory phenotype of infiltrating inflammatory cells in adipose tissue in addition to a reduction in total adipose tissue volume in response to exercise $[4,5,8,40]$.

Neopterin and KTR are indirect measures of IFN- $\gamma$ activity, which plays a central role in activating cellular immune response [19, 43]. Neopterin and KTR have been found to be associated with future major vascular events and all-cause mortality [11]. We found a significant association between sedentary behaviour and neopterin, but not KTR. A less convincing association between sedentary behaviour and biomarkers associated with IFN- $\gamma$ mediated inflammation might be explained by findings from the above-mentioned intervention study by Noz et al., where increased walking time altered innate immune function towards a less pro-inflammatory state in 


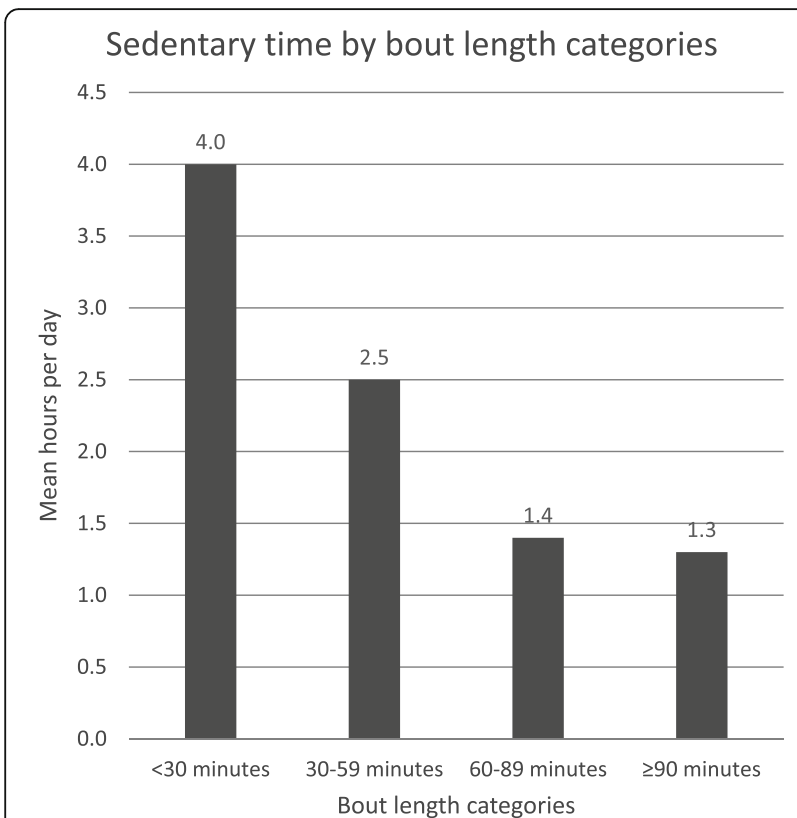

Fig. 2 Mean distribution of daytime sedentary time (from 08:00 am to $10: 00 \mathrm{pm}$ )

combination with an increased IFN- $\gamma$ production capacity upon stimulation [1]. The implication of this in the context of inflammation and vascular disease, is not clear. This is the first study to investigate sedentary behaviour and its association with neopterin and KTR using objective measures for monitoring daytime activity. Prior investigations were predominantly based on questionnaire information and investigated the association to physical activity rather than to sedentary behaviour. The results were inconclusive [21, 22, 31].

We found an inverse association between KA and sedentary behaviour, and the association was strengthened after adjustment for inflammation. KA is metabolised from kynurenine by kynurenine amino transferases (KATs) [27] and is part of a negative feedback loop where chronic inflammation induces immune tolerance via the aryl hydrocarbon receptor (AhR) and the Gprotein-coupled receptor 35 [18, 26, 27, 44, 45]. In a recent review, Joisten et al. argued for the relevance of KA in disease prevention [26]. KAT expression and activity has been found to be induced by chronic exercise via peroxisome proliferator-activated receptor-gamma coactivator 1 alpha $(\mathrm{PGC1} 1 \alpha)$ and the associated transcription factor, peroxisome proliferator activated receptor alpha (PPAR $\alpha$ ) [28-31]. The inverse relationship found in our study suggests a reduced KAT expression during sedentary behaviour. PPAR $\alpha$ has been found to be negatively associated with IL-6 in older adults, and Lustgarten et al. observed that PPAR $\alpha$ activation was related to decreased inflammation [12]. Kynurenic acid could be a molecular link between sedentary behaviour, inflammation, and vascular diseases such as stroke. The KA association was significant only after adjustment for kidney function. KA is eliminated in urine, and lower muscle mass is associated with lower creatinine and higher levels of sedentary

Table 2 The association between biomarkers and total sedentary time

\begin{tabular}{|c|c|c|c|c|c|c|}
\hline & Crud & & & Adjust & & \\
\hline & $\bar{\beta}$ & $95 \% \mathrm{Cl}$ & $P$ & $\bar{\beta}$ & $95 \% \mathrm{Cl}$ & $\mathrm{P}$ \\
\hline hsCRP & & & & & & $R^{2}=0.16$ \\
\hline Total sedentary time & 0.32 & $(0.18,0.47)$ & $<0.001$ & 0.25 & $(0.09,0.39)$ & 0.001 \\
\hline $1 \mathrm{~L}-6^{\mathrm{b}}$ & & & & & & $R^{2}=0.12$ \\
\hline Total sedentary time & 0.26 & $(0.14,0.38)$ & $<0.001$ & 0.17 & $(0.04,0.30)$ & 0.009 \\
\hline $\mid \mathrm{L}-10^{\mathrm{b}}$ & & & & & & $R^{2}=0.04$ \\
\hline Total sedentary time & 0.04 & $(-0.09,0.16)$ & 0.509 & 0.01 & $(-0.12,0.14)$ & 0.841 \\
\hline Neopterin & & & & & & $R^{2}=0.35$ \\
\hline Total sedentary time & 0.24 & $(0.12,0.36)$ & $<0.001$ & 0.12 & $(0.00,0.22)$ & 0.034 \\
\hline PAr-index & & & & & & $R^{2}=0.37$ \\
\hline Total sedentary time & 0.33 & $(0.22,0.45)$ & $<0.001$ & 0.21 & $(0.09,0.30)$ & $<0.001$ \\
\hline KTR & & & & & & $R^{2}=0.45$ \\
\hline Total sedentary time & 0.21 & $(0.09,0.33)$ & 0.001 & 0.06 & $(-0.05,0.15)$ & 0.238 \\
\hline KA & & & & & & $R^{2}=0.48$ \\
\hline Total sedentary time & 0.04 & $(-0.08,0.16)$ & 0.512 & -0.10 & $(-0.21,-0.02)$ & 0.045 \\
\hline
\end{tabular}

hsCRP = high sensitive C-reactive protein. IL-6 = interleukin-6. IL-10 = interleukin-10, PAr-index = (4-pyridoxic acid/(pyridoxal 5'-phosphate + pyridoxal).

$\mathrm{KTR}=$ kynurenine/tryptophan ratio $=($ kynurenine $\mathrm{nM} /$ tryptophan $\mu \mathrm{M}), \mathrm{KA}=$ kynurenic acid

a Model: biomarker is the dependent variable. Sedentary time, age, sex, waist circumference, and creatinine are independent variables

${ }^{\mathrm{b}}$ For IL- 6 and IL-10, Tobit regressions were used for the significance test and regular linear regressions to calculate the beta coefficients 
Table 3 Adjusted multiple regression model of the association between total sedentary time and biomarkers, $n=242$

\begin{tabular}{llll}
\hline Sedentary time & $\boldsymbol{\beta}$ & $\mathbf{9 5 \%} \mathbf{C l}$ & $\mathbf{P}$ \\
\hline IL-6 & 0.12 & $(-0.01,0.24)$ & 0.069 \\
Neopterin & 0.08 & $(-0.07,0.23)$ & 0.280 \\
PAr-index & 0.25 & $(0.10,0.39)$ & 0.001 \\
Kynurenic acid & -0.19 & $(-0.35,-0.03)$ & 0.021 \\
Age & 0.16 & $(0.03,0.29)$ & 0.017 \\
Sex (male) & 0.01 & $(-0.28,0.30)$ & 0.941 \\
Creatinine & 0.02 & $(-0.17,0.21)$ & 0.826 \\
Waist circumference & 0.21 & $(0.08,0.33)$ & 0.002 \\
\hline
\end{tabular}

IL-6 = interleukin-6. PAr-index = (4-pyridoxic acid/(pyridoxal 5'-phosphate + pyridoxal)

behaviour [46]. This can explain the observed change after the adjustments. In the sensitivity analyses restricted to those with a value for hsCRP, the association to KA was no longer significant, probably due to lower sample size. The strengthened association between sedentary behaviour and KA, independent of inflammation in the final model, can be seen as a 'proof of concept' of the connection between sedentary behaviour and immune modulation.

Prior studies have found increased levels of the antiinflammatory cytokine IL-10 in response to exercise [4, 15-17]. A single bout of MVPA has been associated with an increased expression of IL- 6 from the myocytes, and this spike of IL-6 is believed to induce IL-10 [4]. We expected an inverse association between sedentary behaviour and IL-10. The lack of association between sedentary time and IL-10 in our study might be explained by the distinction between physical activity in general (the inverse of sedentary behaviour) and MVPA, which has a higher level of energy expenditure. This also illustrates how duration and intensity of cytokine expression is important for the downstream effects. IL-6 falls

Table 4 Adjusted multiple regression model of the association between total sedentary time and biomarkers restricted to those with a value for hsCRP, $n=161$

\begin{tabular}{llll}
\hline Sedentary time & $\boldsymbol{\beta}$ & $\mathbf{9 5 \%} \mathbf{C l}$ & $\mathbf{P}$ \\
\hline IL-6 & 0.10 & $(-0.06,0.26)$ & 0.229 \\
Neopterin & 0.09 & $(-0.11,0.29)$ & 0.374 \\
PAr-index & 0.31 & $(0.13,0.49)$ & 0.001 \\
Kynurenic acid & -0.17 & $(-0.38,0.04)$ & 0.110 \\
Age & 0.11 & $(-0.05,0.27)$ & 0.195 \\
Sex (male) & 0.05 & $(-0.31,0.40)$ & 0.803 \\
Creatinine & -0.05 & $(-0.30,0.20)$ & 0.692 \\
Waist circumference & 0.15 & $(-0.01,-0.31)$ & 0.062 \\
\hline
\end{tabular}

IL-6 = interleukin-6. PAr-index = (4-pyridoxic acid/(pyridoxal 5'-phosphate + pyridoxal) to basal levels within an hour after exercise [4], and this dual role of IL-6 prompts caution when designing intervention studies.

From our results, it seems that all sedentary behaviour was associated with increased inflammation, but that there was a trend towards stronger associations to sedentary time accumulated through longer bouts. In some cases, the association between sedentary behaviour and the biomarker was no longer significant when stratified for bout length because of reduced statistical power, which also potentially explain the spread in the results.

The strength of this study lies in the sample size, the carefully characterised population of older patients with vascular disease, and the objective measure of sedentary behaviour over several days in a habitual setting. The Nor-COAST population is representative of the group of patients who have suffered mild strokes [34]. The use of both traditional and novel biomarkers has enabled us to confirm prior findings, contributing to filling in the gaps of uncertainties and to opening new and interesting paths and new perspectives. Information about other explanatory variables such as age, waist circumference, and kidney function have increased the validity of the results.

Because of the observational nature of the study, we can only identify associations and not any causal direction. Methods of measuring, defining, and analysing sedentary behaviour were thoroughly discussed in a prior study [47], however, because sedentary bouts were cut if they crossed the day/night timeline, some overrepresentation of shorter bout lengths and concordant underestimation/to the expense of longer bout lengths may have occurred. As shown in the baseline table, the stroke patients in this study were fitter compared to the entire Nor-COAST population, and the findings cannot be generalised to the entire stroke population.

\section{Conclusion}

This novel result indicates that the PAr-index is a potentially useful biomarker in future studies on sedentary behaviour and disease progression. The association to KA opens fresh interesting pathways to understanding disease progression in general, the hazards of sedentary behaviour in particular, and should be further investigated.

\footnotetext{
Abbreviations

AhR: Aryl hydrocarbon receptor; BMI: Body mass index; CRP: C-reactive protein; eGFR: Estimated glomerular filtration rate; hsCRP: High sensitive Creactive protein; IFN-צ: interferon gamma; IL-6: Interleukin-6; IL-

10: Interleukin-10; KA: Kynurenic acid; KAT: Kynurenine aminotransferases; KP: Kynurenine pathway; KTR: Kynurenine/tryptophan ratio; MVPA: Moderate to vigorous physical activity; NIHSS: National Institute of Stroke Scale; NorCOAST: Norwegian Cognitive Impairment After Stroke Study; PArindex: Pyridoxal acid ratio index; PLP: Pyridoxal-5-phosphate; PL: Pyridoxal; PA: Pyridoxal acid; PGC1a: Peroxisome proliferator-activated receptor-gamma coactivator 1 alpha; PPARa: Peroxisome proliferator-activated receptor alpha; REK: Regional ethics committee
} 


\section{Supplementary Information}

The online version contains supplementary material available at https://doi. org/10.1186/s12883-021-02343-0.

Additional file 1: Supplementary Table 1. Crude and adjusted linear regression analyses of the association between biomarkers and time in sedentary behaviour by bout-length category.

\section{Acknowledgements}

The authors appreciate the valuable advice and practical assistance provided by expert personnel at Biobank $1^{\oplus}$, the research biobank of Central Norway, in collection, processing and storage of the biological material.

\section{Authors' contributions}

KNA has planned this study. She has planned analyses, performed the statistical analyses and interpreted the results. She has written the article. TA has contributed to the design of this study and was responsible for the physical activity data from the main study. She has contributed in revising the manuscript. JA has supervised the process of developing and describing the statistical method and contributed in the interpretation and the presentation of the data. TEM has analysed the cytokines used in this study. Also contributed in the post analytical work and defined the method of handling the data and to interpret the results. He has also contributed to revising the manuscript. MN has contributed to the development of the study and revised the manuscript. HN has contributed to the development of the study and revised the manuscript. He has also contributed to the development of the statistical method and the interpreting of the results. IS has designed and lead the work of the main project and contributed in the revision of the manuscript. PMU has been in charge of analysing the biomarkers from Bevital. He has also contributed in the preparation phase of designing the study and selecting the analytic platform and in interpreting the results and the revision of the manuscript. AU has contributed with the statistical analyses and the interpretation of the results. He has contributed with detailed knowledge of the biomarkers from Bevital and to the process of writing the manuscript. ABK has contributed to the development of the study and to the selection of biomarkers and interpretation of the results. She has also contributed to the writing process. All authors have read and agreed to the published version of the manuscript and are accountable for the content.

\section{Funding}

The lead author was granted a PhD-scholarship from the Western Norway Regional Health Authority including funding for the laboratory analyses. The Nor-COAST study was granted 9 million NOKs from the Norwegian Health Association. The funding was granted based on the project description, but the funders does not have an active role in the further scientific process.

\section{Availability of data and materials}

Due to Norwegian regulations and conditions for informed consent, the dataset is not publicly available.

\section{Declarations}

\section{Ethics approval and consent to participate}

This study was conducted in accordance with institutional guidelines and was approved by the Regional Committee of Medical and Health Research Ethics (REK no: 2017/2060/REK Midt, 2015/171/REK Nord). The study was registered at Clinicaltrials.gov (NCT02650531). The participation required that the patient were able to give informed consent. For patients unable to consent for themselves, next of kin may give oral consent.

\section{Consent for publication}

Not applicable. The publication does not contain individual data.

\section{Competing interests}

The authors declare that they have no competing interests.

\section{Author details}

'Institute of Clinical Medicine (K1), University of Bergen, Bergen, Norway.

${ }^{2}$ Department of Internal Medicine, Haraldsplass Deaconess Hospital, Bergen,
Norway. ${ }^{3}$ Department of Neuromedicine and Movement Science, Faculty of Medicine and Health Science, NTNU-Norwegian University of Science and Technology, Trondheim, Norway. ${ }^{4}$ Centre for Clinical Research, Haukeland University Hospital, Bergen, Norway. ${ }^{5}$ Department of Immunology, Oslo University Hospital and University of Oslo, Oslo, Norway. ${ }^{6}$ Research Laboratory, Nordland Hospital, Bodø, and K.G. Jebsen TREC, University of Troms $\varnothing$, Troms $\varnothing$, Norway. ${ }^{7}$ Centre of Molecular Inflammation Research, Norwegian University of Science and Technology, Trondheim, Norway. ${ }^{8}$ Department of Clinical Science (K2), University of Bergen, Bergen, Norway. ${ }^{9}$ Department of Neurology, Haukeland University Hospital, Bergen, Norway.

${ }^{10}$ Centre for age-related medicine, Stavanger University Hospital, Stavanger, Norway. ${ }^{11}$ Department of Geriatrics, Clinic of internal medicine, St Olavs Hospital, Trondheim University Hospital, Trondheim, Norway. ${ }^{12}$ Bevital AS, Bergen, Norway. ${ }^{13}$ Department of Geriatric Medicine, Oslo University Hospital, Ullevaal, Oslo, Norway.

Received: 18 March 2021 Accepted: 26 July 2021

Published online: 16 August 2021

\section{References}

1. Noz MP, Hartman YAW, Hopman MTE, Willems P, Tack CJ, Joosten LAB, et al. Sixteen-week physical activity intervention in subjects with increased Cardiometabolic risk shifts innate immune function towards a less Proinflammatory state. J Am Heart Assoc. 2019;8(21):e013764. https://doi. org/10.1161/JAHA.119.013764.

2. Wirth K, Klenk J, Brefka S, Dallmeier D, Faehling K, Roque IFM, et al. Biomarkers associated with sedentary behaviour in older adults: a systematic review. Ageing Res Rev. 2017;35:87-111. https://doi.org/10.1016/ j.arr.2016.12.002

3. Lavie CJ, Ozemek C, Carbone S, Katzmarzyk PT, Blair SN. Sedentary behavior, exercise, and cardiovascular health. Circ Res. 2019;124(5):799-815. https:// doi.org/10.1161/CIRCRESAHA.118.312669.

4. Gleeson M, Bishop NC, Stensel DJ, Lindley MR, Mastana SS, Nimmo MA. The anti-inflammatory effects of exercise: mechanisms and implications for the prevention and treatment of disease. Nat Rev Immunol. 2011;11(9):607-15. https://doi.org/10.1038/nri3041

5. Tir AMD, Labor M, Plavec D. The effects of physical activity on chronic subclinical systemic inflammation. Arh Hig Rada Toksikol. 2017;68(4):276-86. https://doi.org/10.1515/aiht-2017-68-2965.

6. Tremblay MS, Aubert S, Barnes JD, Saunders TJ, Carson V, Latimer-Cheung $A E$, et al. Sedentary behavior research network (SBRN) - terminology consensus project process and outcome. Int J Behav Nutr Phys Act. 2017; 14(1):75. https://doi.org/10.1186/s12966-017-0525-8.

7. Benatti FB, Ried-Larsen $M$. The effects of breaking up prolonged sitting time: a review of experimental studies. Med Sci Sports Exerc. 2015;47(10):2053-61. https://doi.org/10.1249/MSS.0000000000000654.

8. Parsons TJ, Sartini C, Welsh P, Sattar N, Ash S, Lennon LT, et al. Physical activity, sedentary behavior, and inflammatory and hemostatic markers in men. Med Sci Sports Exerc. 2017;49(3):459-65. https://doi.org/10.1249/MSS. 0000000000001113.

9. Dunstan DW, Thorp AA, Healy GN. Prolonged sitting: is it a distinct coronary heart disease risk factor? Curr Opin Cardiol. 2011;26(5):412-9. https://doi. org/10.1097/HCO.0b013e3283496605.

10. Ridker PM. From C-reactive protein to Interleukin-6 to Interleukin-1: moving upstream to identify novel targets for Atheroprotection. Circ Res. 2016; 118(1):145-56. https://doi.org/10.1161/CIRCRESAHA.115.306656.

11. Pedersen ER, Midttun O, Ueland PM, Schartum-Hansen H, Seifert R, Igland J, et al. Systemic markers of interferon-gamma-mediated immune activation and long-term prognosis in patients with stable coronary artery disease. Arterioscler Thromb Vasc Biol. 2011;31(3):698-704. https://doi.org/10.1161/A TVBAHA.110.219329.

12. Lustgarten MS, Fielding RA. Metabolites associated with circulating Interleukin-6 in older adults. J Gerontol A Biol Sci Med Sci. 2017;72(9):127783.

13. Ferrucci L, Gonzalez-Freire M, Fabbri E, Simonsick E, Tanaka T, Moore Z, et al. Measuring biological aging in humans: a quest. Aging Cell. 2020;19(2): e13080. https://doi.org/10.1111/acel.13080.

14. Henson J, Yates T, Edwardson CL, Khunti K, Talbot D, Gray L, et al. Sedentary time and markers of chronic low-grade inflammation in a high risk population. PLoS One. 2013;8(10):e78350. https://doi.org/10.1371/journal. pone.0078350. 
15. Leal LG, Lopes MA, Batista ML Jr. Physical exercise-induced Myokines and muscle-adipose tissue crosstalk: a review of current knowledge and the implications for health and metabolic diseases. Front Physiol. 2018;9:1307. https://doi.org/10.3389/fphys.2018.01307.

16. Abd El-Kader SM, Al-Jiffri OH. Aerobic exercise modulates cytokine profile and sleep quality in elderly. Afr Health Sci. 2019;19(2):2198-207. https://doi. org/10.4314/ahs.v19i2.45.

17. Sallam N, Laher I. Exercise modulates oxidative stress and inflammation in aging and cardiovascular diseases. Oxidative Med Cell Longev. 2016;2016: 7239639.

18. Mandi $Y$, Vecsei L. The kynurenine system and immunoregulation. J Neural Transm (Vienna). 2012;119(2):197-209. https://doi.org/10.1007/s00702-0110681-y.

19. Schrocksnadel K, Wirleitner B, Winkler C, Fuchs D. Monitoring tryptophan metabolism in chronic immune activation. Clin Chim Acta. 2006;364(1-2): 82-90. https://doi.org/10.1016/j.cca.2005.06.013.

20. Eussen SJ, Ueland PM, Vollset SE, Nygard O, Midttun O, Sulo G, et al. Kynurenines as predictors of acute coronary events in the Hordaland health study. Int J Cardiol. 2015;189:18-24. https://doi.org/10.1016/j.ijcard.2015. 03.413 .

21. Valdiglesias V, Sanchez-Flores M, Maseda A, Lorenzo-Lopez L, Marcos-Perez D, Lopez-Corton A, et al. Immune biomarkers in older adults: role of physical activity. J Toxicol Environ Health A. 2017;80(13-15):605-20. https:// doi.org/10.1080/15287394.2017.1286898.

22. Theofylaktopoulou D, Midttun O, Ulvik A, Ueland PM, Tell GS, Vollset SE, et al. A community-based study on determinants of circulating markers of cellular immune activation and kynurenines: the Hordaland health study. Clin Exp Immunol. 2013;173(1):121-30. https://doi.org/10.1111/cei.12092.

23. Ulvik A, Midttun O, Pedersen ER, Eussen SJ, Nygard O, Ueland PM. Evidence for increased catabolism of vitamin B-6 during systemic inflammation. Am J Clin Nutr. 2014;100(1):250-5. https://doi.org/10.3945/ajcn.114.083196.

24. Ulvik A, Midttun O, Pedersen ER, Nygard O, Ueland PM. Association of plasma B-6 vitamers with systemic markers of inflammation before and after pyridoxine treatment in patients with stable angina pectoris. Am J Clin Nutr. 2012;95(5):1072-8. https://doi.org/10.3945/ajcn.111.029751.

25. Zuo H, Tell GS, Ueland PM, Nygard O, Vollset SE, Midttun O, et al. The PAr index, an indicator reflecting altered vitamin B-6 homeostasis, is associated with long-term risk of stroke in the general population: the Hordaland health study (HUSK). Am J Clin Nutr. 2018;107(1):105-12. https://doi.org/10.1 093/ajcn/nqx012.

26. Joisten N, Walzik D, Metcalfe AJ, Bloch W, Zimmer P. Physical exercise as kynurenine pathway modulator in chronic diseases: implications for immune and energy homeostasis. Int J Tryptophan Res. 2020;13: 1178646920938688.

27. Wirthgen E, Hoeflich A, Rebl A, Gunther J. Kynurenic acid: the Janus-faced role of an immunomodulatory tryptophan metabolite and its link to pathological conditions. Front Immunol. 2017:8:1957.

28. Agudelo LZ, Femenia T, Orhan F, Porsmyr-Palmertz M, Goiny M, MartinezRedondo V, et al. Skeletal muscle PGC-1alpha1 modulates kynurenine metabolism and mediates resilience to stress-induced depression. Cell. 2014; 159(1):33-45. https://doi.org/10.1016/j.cell.2014.07.051.

29. Schlittler M, Goiny M, Agudelo LZ, Venckunas T, Brazaitis M, Skurvydas A, et al. Endurance exercise increases skeletal muscle kynurenine aminotransferases and plasma kynurenic acid in humans. Am J Physiol Cell Physiol. 2016;310(10):C836-40. https://doi.org/10.1152/ajpcell.00053.2016.

30. Cervenka I, Agudelo LZ, Ruas JL. Kynurenines: Tryptophan's metabolites in exercise, inflammation, and mental health. Science. 2017;357(6349):eaaf9794.

31. Metcalfe AJ, Koliamitra C, Javelle F, Bloch W, Zimmer P. Acute and chronic effects of exercise on the kynurenine pathway in humans - a brief review and future perspectives. Physiol Behav. 2018;194:583-7. https://doi.org/10.1 016/j.physbeh.2018.07.015.

32. Baumgartner R, Berg M, Matic L, Polyzos KP, Forteza MJ, Hjorth SA, et al. Evidence that a deviation in the kynurenine pathway aggravates atherosclerotic disease in humans. J Intern Med. 2021;289(1):53-68. https:// doi.org/10.1111/joim.13142.

33. Thingstad P, Askim T, Beyer MK, Brathen G, Ellekjaer $\mathrm{H}$, Ihle-Hansen $\mathrm{H}$, et al The Norwegian cognitive impairment after stroke study (nor-COAST): study protocol of a multicentre, prospective cohort study. BMC Neurol. 2018;18(1): 193. https://doi.org/10.1186/s12883-018-1198-x.

34. Kuvas KR, Saltvedt I, Aam S, Thingstad P, Ellekjaer H, Askim T. The risk of selection Bias in a clinical multi-center cohort study. Results from the
Norwegian cognitive impairment after stroke (nor-COAST) study. Clin Epidemiol. 2020;12:1327-36. https://doi.org/10.2147/CLEP.S276631.

35. Brott T, Adams HP Jr, Olinger CP, Marler JR, Barsan WG, Biller J, et al. Measurements of acute cerebral infarction: a clinical examination scale. Stroke. 1989;20(7):864-70. https://doi.org/10.1161/01.STR.20.7.864.

36. Duffy L, Gajree S, Langhorne P, Stott DJ, Quinn TJ. Reliability (inter-rater agreement) of the Barthel index for assessment of stroke survivors: systematic review and meta-analysis. Stroke. 2013;44(2):462-8. https://doi. org/10.1161/STROKEAHA.112.678615.

37. Banks JL, Marotta CA. Outcomes validity and reliability of the modified Rankin scale: implications for stroke clinical trials: a literature review and synthesis. Stroke. 2007;38(3):1091-6. https://doi.org/10.1161/01.STR.00002583 55.23810.c6.

38. Stockmann H, Neves AA, Stairs S, Ireland-Zecchini H, Brindle KM, Leeper FJ. Development and evaluation of new cyclooctynes for cell surface glycan imaging in cancer cells. Chem Sci. 2011;2(5):932-6. https://doi.org/10.1039/ c0sc00631a.

39. Sorgdrager FJH, Naude PJW, Kema IP, Nollen EA, Deyn PP. Tryptophan metabolism in Inflammaging: from biomarker to therapeutic target. Front Immunol. 2019;10:2565. https://doi.org/10.3389/fimmu.2019.02565.

40. Cronin O, Keohane DM, Molloy MG, Shanahan F. The effect of exercise interventions on inflammatory biomarkers in healthy, physically inactive subjects: a systematic review. QJM. 2017;110(10):629-37. https://doi.org/10.1 093/qjmed/hcx091.

41. Wang W, Griswold ME. Estimating overall exposure effects for the clustered and censored outcome using random effect Tobit regression models. Stat Med. 2016;35(27):4948-60. https://doi.org/10.1002/sim.7045.

42. Swindell N, Rees P, Fogelholm M, Drummen M, MacDonald I, Martinez JA, et al. Compositional analysis of the associations between 24-h movement behaviours and cardio-metabolic risk factors in overweight and obese adults with pre-diabetes from the PREVIEW study: cross-sectional baseline analysis. Int J Behav Nutr Phys Act. 2020;17(1):29. https://doi.org/10.1186/s12 966-020-00936-5.

43. Teixeira LK, Fonseca BP, Barboza BA, Viola JP. The role of interferon-gamma on immune and allergic responses. Mem Inst Oswaldo Cruz. 2005;100(Suppl 1):137-44. https://doi.org/10.1590/S0074-02762005000900024.

44. Tanaka T, Narazaki M, Kishimoto T. IL-6 in inflammation, immunity, and disease. Cold Spring Harb Perspect Biol. 2014;6(10):a016295. https://doi.org/1 0.1101/cshperspect.a016295

45. Jung TW, Park J, Sun JL, Ahn SH, Abd El-Aty AM, Hacimuftuoglu A, et al. Administration of kynurenic acid reduces hyperlipidemia-induced inflammation and insulin resistance in skeletal muscle and adipocytes. Mol Cell Endocrinol. 2020;518:110928. https://doi.org/10.1016/j.mce.2020.110928.

46. Smith L, Tully M, Jacob L, Blackburn N, Adlakha D, Caserotti P, et al. The Association Between Sedentary Behavior and Sarcopenia Among Adults Aged $>/=65$ Years in Low- and Middle-Income Countries. Int J Environ Res Public Health. 2020;17(5):1708.

47. Alme KN, Knapskog AB, Naess $H$, Naik M, Beyer $M$, Ellekjaer $H$, et al. Is longbout sedentary behaviour associated with long-term glucose levels 3 months after acute ischaemic stroke? A prospective observational cohort study. BMJ Open. 2020;10(11):e037475. https://doi.org/10.1136/bmjopen-202 0-037475

\section{Publisher's Note}

Springer Nature remains neutral with regard to jurisdictional claims in published maps and institutional affiliations.

Ready to submit your research? Choose BMC and benefit from:

- fast, convenient online submission

- thorough peer review by experienced researchers in your field

- rapid publication on acceptance

- support for research data, including large and complex data types

- gold Open Access which fosters wider collaboration and increased citations

- maximum visibility for your research: over $100 \mathrm{M}$ website views per year

At $\mathrm{BMC}$, research is always in progress.

Learn more biomedcentral.com/submissions 